\title{
Unexpected Features of Supersymmetry with Central Charges
}

\author{
Rutwig Campoamor-Stursberg $\dagger$ \\ $\dagger$ I.M.I-U.C.M, Plaza de Ciencias 3, E-28040 Madrid, Spain \\ E-mail: rutwig@pdi.ucm.es
}

Michel Rausch de Traubenberg

$\ddagger$ IPHC-DRS, UdS, CNRS, IN2P3, 23 rue du Loess, F-67037 Strasbourg Cedex, France

E-mail: Michel.Rausch@IReS.in2p3.fr

\begin{abstract}
It is shown that $N=2$ supersymmetric theories with central charges present some hidden quartic symmetry. This enables us to construct representations of the quartic structure induced by superalgebra representations.
\end{abstract}

PACS numbers: $11.30 \mathrm{~Pb}, 02.20 \mathrm{~Sv}$ 


\section{Motivation and introduction}

For a long time, Lie algebras and superalgebras have been the main group-theoretical tool for the description of physical phenomena, leading to the belief that binary structures are the only adequate algebraic tool capable of reflecting a realistic description of processes in physics. However, evolution of the theoretical background and more recent formal developments and, in particular, efforts towards a unified and effective description of interactions has shown that it is convenient, if not unavoidable, to consider algebras beyond the binary structures. Examples of this generalized algebraic approach are given e.g. by the $n$-linear algebras in Quantum Mechanics [1], ternary algebras in the description of multiple $M_{2}$-branes [2, 3] or higher order extensions of the Poincaré algebra [4].

Among these possibilities, the $F$-ary extensions $(F>2)$ of Lie superalgebras (called Lie algebras of order $F$ ) introduced and analysed in [4, 5, 6, 7], offered the possibility of defining higher order extensions of the Poincaré algebra based on solid physical arguments. In particular, a specific cubic extension in arbitrary space-time dimensions was shown to be of interest in the frame of Quantum Field Theory [8, 9, 10]. In addition, it was possible to conceive and construct the notions of group [6] and adapted superspace associated to these structures [11].

In this work we show that certain graded Lie superalgebras might induce specific quartic extensions of Lie algebras. This construction is then applied to show that we can associate naturally a quartic extension of the Poincaré algebra to the standard $N=2$ supersymmetric extensions (with central charges). In this construction, the central charges turn out to play a key role, as they constitute the essential ingredient to introduce the notion of a hidden quartic symmetry. It follows that massive invariant $N=2$ Lagrangians are also invariant with respect to these hidden symmetries. We finally conclude that the quartic extensions obtained by this method give rise to a hierarchy of representations that emerges from the standard representation of the corresponding supersymmetric theory.

\section{Quartic structures induced by quadratic algebras}

The $F$-order structures were introduced mainly with the purpose of providing new nontrivial higher order (order greater that two) extensions of the Poincaré algebra involving generators $Q$ which can be seen as "an $F$-root" of the space-time translations (see e.g. (2.4) or (2.5) when $F=4)$ 4]. In this work we are mainly interested in the quartic case. Consequently, we briefly recall the salient properties of these structures. 


\subsection{Quartic extensions of the Poincaré algebra}

The vector space $\mathfrak{g}=\mathfrak{g}_{0} \oplus \mathfrak{g}_{1}=\left\langle X_{i}, i=1, \cdots, \operatorname{dim} \mathfrak{g}_{0}\right\rangle \oplus\left\langle Y_{a}, a=1, \cdots, \operatorname{dim} \mathfrak{g}_{1}\right\rangle \dot{\dagger}$ is called an elementary Lie algebra of order four if it satisfies the following brackets

$$
\begin{aligned}
& {\left[X_{i}, X_{j}\right]=f_{i j}{ }^{k} X_{k}, \quad\left[X_{i}, Y_{a}\right]=R_{i a}{ }^{b} Y_{b},} \\
& \left\{Y_{a_{i}}, Y_{a_{2}}, Y_{a_{3}}, Y_{a_{4}}\right\}=\sum_{\sigma \in S_{4}} Y_{\sigma\left(a_{1}\right)} Y_{\sigma\left(a_{2}\right)} Y_{\sigma\left(a_{3}\right)} Y_{\sigma\left(a_{4}\right)}=Q_{a_{1} a_{2} a_{3} a_{4}}{ }^{i} X_{i},
\end{aligned}
$$

$S_{4}$ being the permutation group with four elements. In addition, we have also the following generalised Jacobi identities:

$$
\begin{aligned}
& {\left[Y_{a_{1}},\left\{Y_{a_{2}}, Y_{a_{3}}, Y_{a_{4}}, Y_{a_{5}}\right\}\right]+\left[Y_{a_{2}},\left\{Y_{a_{3}}, Y_{a_{4}}, Y_{a_{5}}, Y_{a_{1}}\right\}\right]+\left[Y_{a_{3}},\left\{Y_{a_{4}}, Y_{a_{5}}, Y_{a_{1}}, Y_{a_{2}}\right\}\right]+} \\
& {\left[Y_{a_{4}},\left\{Y_{a_{5}}, Y_{a_{1}}, Y_{a_{2}}, Y_{a_{3}}\right\}\right]+\left[Y_{a_{5}},\left\{Y_{a_{1}}, Y_{a_{2}}, Y_{a_{3}}, Y_{a_{4}}\right\}\right]=0 .}
\end{aligned}
$$

Along the lines of the algebraic structure (2.1), there is the possibility of constructing quartic extensions of the Poincaré algebra in arbitrary space-time dimensions. Within this frame, two quartic extensions of the Poincaré algebra will be considered in detail: that in $D=4$ for obvious physical reasons, as well as $D=10$, which will be shown later (Section 3) to constitute an exceptional case.

The quartic extensions of the Poincaré algebra in $D=4$ dimensions are constructed by considering two Majorana spinord $\mathcal{S}$. In the $\mathfrak{s l}(2, \mathbb{C}) \cong \mathfrak{s o}(1,3)$ notations of dotted and undotted indices, a left-handed spinor is given by $\psi_{L}{ }^{\alpha}$ and a right-handed spinor by $\bar{\psi}_{R \dot{\alpha}}$. The spinor conventions to raise/lower indices are as follows $\psi_{L \alpha}=\varepsilon_{\alpha \beta} \psi_{L}{ }^{\beta}$, $\psi_{L}{ }^{\alpha}=\varepsilon^{\alpha \beta} \psi_{L \beta}, \bar{\psi}_{R \dot{\alpha}}=\varepsilon_{\dot{\alpha} \dot{\beta}} \bar{\psi}_{R}^{\dot{\beta}}, \bar{\psi}_{R}^{\dot{\alpha}}=\varepsilon^{\dot{\alpha} \dot{\beta}} \bar{\psi}_{R \dot{\beta}}$ with $\left(\psi_{\alpha}\right)^{\star}=\bar{\psi}_{\dot{\alpha}}, \varepsilon_{12}=\varepsilon_{\dot{1} \dot{2}}=1$, $\varepsilon^{12}=\varepsilon^{i \dot{2}}=-1$. The $4 D$ Dirac matrices, in the Weyl representation, are

$$
\Gamma^{\mu}=\left(\begin{array}{cc}
0 & \sigma^{\mu} \\
\bar{\sigma}^{\mu} & 0
\end{array}\right),
$$

with $\sigma_{\alpha \dot{\alpha}}^{\mu}=\left(1, \sigma^{i}\right), \bar{\sigma}^{\mu \dot{\alpha} \alpha}=\left(1,-\sigma^{i}\right), \sigma^{i}(i=1,2,3)$ being the Pauli matrices. With these notations, we introduce two series of Majorana spinors $Q^{I}{ }_{\alpha}, \bar{Q}_{I \dot{\alpha}}$ satisfying the relation $\left(Q^{I}{ }_{\alpha}\right)^{\dagger}=\bar{Q}_{I \dot{\alpha}}$. The Lie algebra of order four with $\mathfrak{g}_{0}=I \mathfrak{s o}(1,3)$ (the Poincaré algebra) and $\mathfrak{g}_{1}=\left\langle Q^{I}{ }_{\alpha}, \bar{Q}_{I \dot{\alpha}}\right\rangle$ define the following quartic extension of the Poincaré algebra\|

$$
\begin{aligned}
& \left\{Q_{\alpha_{1}}^{I_{1}}, Q^{I_{2}}{ }_{\alpha_{2}}, Q^{I_{3}}{ }_{\alpha_{3}}, Q^{I_{4}}{ }_{\alpha_{4}}\right\}=0 \\
& \left\{Q^{I_{1}}{ }_{\alpha_{1}}, Q^{I_{2}{ }_{\alpha_{2}}}, Q^{I_{3}}{ }_{\alpha_{3}}, \bar{Q}_{I_{4} \dot{\alpha}_{4}}\right\}=2 i\left(\delta_{{ }_{I_{4}}}^{I_{1}} \varepsilon^{I_{2} I_{3}} \varepsilon_{\alpha_{2} \alpha_{3}} \sigma_{\alpha_{1} \dot{\alpha}_{4}}+\delta_{I_{4}}^{I_{2}} \varepsilon^{I_{1} I_{3}} \varepsilon_{\alpha_{1} \alpha_{3}} \sigma_{\alpha_{2} \dot{\alpha}_{4}}^{\mu}\right.
\end{aligned}
$$

$\ddagger$ We would like to point out that the symbol $\oplus$ here and in the sequel can be understood as a direct sum of vector spaces.

$\S$ For a brief summary of the properties of spinors, see section 3.1

\| For shortness, we only give the quartic brackets explicitly. 


$$
\begin{aligned}
& \left.+\delta^{I_{3}} \varepsilon_{4} \varepsilon^{I_{1} I_{2}} \varepsilon_{\alpha_{1} \alpha_{2}} \sigma_{\alpha_{3} \dot{\alpha}_{4}}^{\mu}\right) P_{\mu}, \\
\left\{Q^{I_{1}}{ }_{\alpha_{1}}, Q^{I_{2}}{ }_{\alpha_{2}}, \bar{Q}_{I_{3} \dot{\alpha}_{3}}, \bar{Q}_{I_{4} \dot{\alpha}_{4}}\right\} & =0,
\end{aligned}
$$

the remaining brackets involving three $\bar{Q}$ and one $Q$ or four $\bar{Q}$ being obtained immediately (the tensor $\varepsilon^{I J}$ is defined by $-\varepsilon^{12}=\varepsilon^{21}=\varepsilon_{12}=-\varepsilon_{21}=1$ ).

The quartic extension of the Poincaré algebra in $D=10$ is constructed by considering a Majorana spinor $Q_{A}\left(\mathfrak{g}_{0}=I \mathfrak{s o}(1,9)\right.$ and $\left.\mathfrak{g}_{1}=\left\langle Q_{A}\right\rangle\right)$. We denote $A=\left(a, a^{\prime}\right)$ the spinor indices, with $a$ (resp. $\left.a^{\prime}\right)$ representing the indices for the left(resp. right-)handed part of the spinor $Q_{A}$. With these conventions, we introduce $Q_{a}^{+}$ the left-handed and $Q_{a^{\prime}}^{-}$the right handed part of $Q$, so that the quartic part of the algebra takes the form

$\left\{Q_{a_{1}}^{+}, Q_{a_{2}}^{+}, Q_{a_{3}}^{+}, Q_{a_{4}}^{+}\right\}=0$

$\left\{Q_{a_{1}}^{+}, Q_{a_{2}}^{+}, Q_{a_{3}}^{+}, Q_{a_{4}^{\prime}}^{-}\right\}=2 i\left(C_{a_{1} a_{4}^{\prime}}^{+-}\left(\Sigma^{\mu} C^{-+}\right)_{a_{2} a_{3}}+C_{a_{2} a_{4}^{\prime}}^{+-}\left(\Sigma^{\mu} C^{-+}\right)_{a_{1} a_{3}}+C_{a_{3} a_{4}^{\prime}}^{+-}\left(\Sigma^{\mu} C^{-+}\right)_{a_{1} a_{2}}\right) P_{\mu}$,

$\left\{Q_{a_{1}}^{+}, Q_{a_{2}}^{+}, Q_{a_{3}^{\prime}}^{-}, Q_{a_{4}^{\prime}}^{-}\right\}=0$,

with

$$
\Gamma^{\mu}=\left(\begin{array}{cc}
0 & \Sigma^{\mu} \\
\tilde{\Sigma}^{\mu} & 0
\end{array}\right), \quad C=\left(\begin{array}{cc}
0 & C^{+-} \\
C^{-+} & 0
\end{array}\right),
$$

being the 10-dimensional Dirac and the charge conjugation matrices respectively. It has to be emphasized that the algebraic structure defined in this manner is neither an algebra nor a 4-algebra in the usual sense, but a kind of hybrid structure. Indeed, some of the brackets are quadratic $\left[\mathfrak{g}_{0}, \mathfrak{g}_{0}\right] \subseteq \mathfrak{g}_{0},\left[\mathfrak{g}_{0}, \mathfrak{g}_{1}\right] \subseteq \mathfrak{g}_{1}$, while some others are quartic $\left\{\mathfrak{g}_{1}, \mathfrak{g}_{1}, \mathfrak{g}_{1}, \mathfrak{g}_{1}\right\} \subseteq \mathfrak{g}_{0}$. This feature represents one of the difficulties to handle with these algebraic structures. Our aim is to analyse to which extent the quartic bracket given in (2.1) can be obtained by means of appropriate quadratic brackets. It is worthy to be remarked that various attempts to inspect the same problem for cubic instead of quartic extensions did not succeed. The obstructions encountered may provide, in some sense, a structural explanation for the difficulties found in the various constructions of the cubic extensions of the Poincaré algebra, despite of some interesting results obtained with such cubic structures.

\subsection{Relationship between quartic algebras and Lie superalgebras}

The study of relationships between quadratic and higher order algebras is certainly not a new problem. For instance, it has already been established in [13] that some ternary algebras of the Filippov type considered in the Bagger-Lambert-Gustavsson model are equivalent to certain Lie (super-)algebras [2, 12, 13].

We will prove that a similar result can be obtained in this case and that certain types of graded-Lie superalgebras actually induce a quartic structure in analogy to those 
given in (2.1), but with slight formal differences. This will enable us to develop a procedure to associate quartic algebras to binary algebras. There is a natural comparison of this construction and the induction theorem of the second paper of [4] that allows to construct higher order Lie algebras starting from arbitrary Lie (super)algebras. The main difference with respect to this approach is that in the present ansatz higher order brackets are compatible with quadratic brackets.

The starting point for our construction is a $\mathbb{Z}_{2} \times \mathbb{Z}_{2}$-graded Lie superalgebra

$$
\mathfrak{g}=\left(\mathfrak{g}_{(0,0)} \oplus \mathfrak{g}_{(1,1)}\right) \oplus\left(\mathfrak{g}_{(1,0)} \oplus \mathfrak{g}_{(0,1)}\right),
$$

where $(a, b) \in \mathbb{Z}_{2} \times \mathbb{Z}_{2}$ and $\mathfrak{g}_{(a, b)}$ is even (resp. odd) when $a+b=0 \bmod 2$ (resp. $a+b=1 \bmod 2)$. In the following $\mathfrak{g}_{0}=\mathfrak{g}_{(0,0)} \oplus \mathfrak{g}_{(1,1)}\left(\operatorname{resp} . \quad \mathfrak{g}_{1}=\mathfrak{g}_{(0,1)} \oplus \mathfrak{g}_{(1,0)}\right)$ will always denote the even (resp. odd) sector of the algebra. Considering the corresponding bases for the grading blocks:

$$
\begin{array}{ll}
\mathfrak{g}_{(0,0)}=\left\langle B_{i}, i=1, \cdots, \operatorname{dim} \mathfrak{g}_{(0,0)}\right\rangle, & \mathfrak{g}_{(1,1)}=\langle Z\rangle, \\
\mathfrak{g}_{(1,0)}=\left\langle F_{a}^{+}, a=1, \cdots, \operatorname{dim} \mathfrak{g}_{(1,0)}\right\rangle, & \mathfrak{g}_{(0,1)}=\left\langle F_{a}^{-}, a=1, \cdots, \operatorname{dim} \mathfrak{g}_{(0,1)}\right\rangle,
\end{array}
$$

the corresponding commutation relations are

$$
\begin{aligned}
& {\left[B_{i}, B_{j}\right]=f_{i j}^{k} B_{k}, \quad\left[B_{i}, Z\right]=0,} \\
& {\left[B_{i}, F_{a}^{\varepsilon}\right]=R_{i a}^{\varepsilon}{ }^{b} F_{b}^{\varepsilon}, \quad\left[Z, F_{a}^{\varepsilon}\right]=0,} \\
& \left\{F_{i}^{\varepsilon}, F_{j}^{\varepsilon}\right\}=Q_{i j}^{\varepsilon}{ }^{a} B_{a}, \quad\left\{F_{i}^{+}, F_{j}^{-}\right\}=g_{i j} Z,
\end{aligned}
$$

where $\varepsilon= \pm$. Furthermore, the superalgebra defined by (2.8) satisfies also the appropriate Jacobi identities (that we do not recall here). It is important to notice that $\mathfrak{g}_{(1,1)}$ commutes with all remaining factors, in other words, that $Z$ acts like a central charge. Formally, there is no difficulty to generalise this structure to have a higher number of central charges $Z_{i}, i=1, \cdots, k$, so that $\mathfrak{g}_{(1,1)}=\left\langle Z_{1}, \cdots, Z_{k}\right\rangle$. In this work, however, we focus on the applications of the construction to the case of $N=2$ supersymmetric extensions of the Poincaré algebra, so that only one central charge is required. In the following, we only consider the case where $\mathfrak{g}_{(1,1)}$ is of dimension one. On the other hand, we could have considered the following grading blocks: $\mathfrak{g}_{(0,0)}=\langle Z\rangle, \mathfrak{g}_{(1,1)}=\left\langle B_{i}, i=1, \cdots, \operatorname{dim} \mathfrak{g}_{(1,1)}\right\rangle$. In this case, the fermionic part of the brackets reads

$$
\left\{F_{i}^{+}, F_{j}^{-}\right\}=Q_{i j}{ }^{a} B_{a},\left\{F_{i}^{\varepsilon}, F_{j}^{\varepsilon}\right\}=g_{i j}^{\varepsilon} Z, \quad \varepsilon= \pm .
$$

With these preliminary assumptions, we are in situation of establishing the main result of this section by explicitly constructing a quartic algebra associated to the superalgebra (2.7). This structure, in the light of equation (2.1), enables us to express the non-graded part of the algebra in terms of the graded part. In fact, using the obvious relation,

$$
\left\{A_{1}, A_{2}, A_{3}, A_{4}\right\}=\left\{\left\{A_{1}, A_{2}\right\},\left\{A_{3}, A_{4}\right\}\right\}+\left\{\left\{A_{1}, A_{3}\right\},\left\{A_{2}, A_{4}\right\}\right\}+\left\{\left\{A_{1}, A_{4}\right\},\left\{A_{2}, A_{3}\right\}\right\},
$$

the relations

$\left\{F_{a_{1}}^{+}, F_{a_{2}}^{+}, F_{a_{3}}^{+}, F_{a_{4}}^{+}\right\}=\left(Q_{a_{1} a_{2}}^{+}{ }^{i} Q_{a_{3} a_{4}}^{+{ }^{j}}+Q_{a_{1} a_{3}}^{+}{ }^{i} Q_{a_{4} a_{2}}^{+j}+Q_{a_{1} a_{4}}^{+}{ }^{i} Q_{a_{2} a_{3}}^{+}{ }^{j}\right)\left\{B_{i}, B_{j}\right\}$ 
$\left\{F_{a_{1}}^{+}, F_{a_{2}}^{+}, F_{a_{3}}^{+}, F_{a_{4}}^{-}\right\}=2 Z\left(g_{a_{1} a_{4}} Q_{a_{2} a_{3}}^{+}{ }^{i}+g_{a_{2} a_{4}} Q_{a_{1} a_{3}}^{+}{ }^{i}+g_{a_{3} a_{4}} Q_{a_{1} a_{2}}^{+}{ }^{i}\right) B_{i}$ $\left\{F_{a_{1}}^{+}, F_{a_{2}}^{+}, F_{a_{3}}^{-}, F_{a_{4}}^{-}\right\}=Q_{a_{1} a_{2}}^{+}{ }^{i} Q_{a_{3} a_{4}}^{-}{ }^{j}\left\{B_{i}, B_{j}\right\}+2\left(g_{i_{1} i_{3}} g_{i_{2} i_{4}}+g_{i_{1} i_{4}} g_{i_{2} i_{3}}\right) Z^{2}$,

(plus similar relations involving either three $F^{-}$and one $F^{+}$or four $F^{-}$) follow at once. Had we chosen the alternative possibility for the fermionic brackets given above, the brackets (2.10) would be subjected to a corresponding minor modification.

Some comments are in order here. The superalgebra reproduces the algebra of type (2.1), but in a slightly modified form. Since we are constructing an analogue of the four-Lie algebra (2.1), we also assume that the algebra associated to the Lie superalgebra (2.8) inherits the same algebraic structure. More precisely, we suppose that the algebra is partially quadratic and partially quartic, that is, $\left[\mathfrak{g}_{0}, \mathfrak{g}_{0}\right] \subseteq \mathfrak{g}_{0},\left[\mathfrak{g}_{0}, \mathfrak{g}_{1}\right] \subseteq \mathfrak{g}_{1}$ (these brackets are the same of the corresponding brackets of the Lie superalgebra), but now the quartic brackets $\left\{\mathfrak{g}_{1}, \mathfrak{g}_{1}, \mathfrak{g}_{1}, \mathfrak{g}_{1}\right\}$ close quadratically in $\mathfrak{g}_{0}$. This means that the structure which emerges in this process closes in the universal enveloping algebra of $\mathfrak{g}_{0}$ since the R.H.S. involves symmetric products of elements of $\mathfrak{g}_{0}$. For this reason it could be called a non-linear (or quadratic) Lie algebra of order four. To round off the relationship between Lie superalgebras and the algebra defined by the relations (2.10), we have to check that the Jacobi identities of Lie superalgebras reproduce the generalised Jacobi identity (2.2). We observe that there is no need for this to hold in full generality, as there is no reason for the relations (2.8), together with the Jacobi identities of Lie superalgebras, to imply the identity (2.2). However, it happens that if we have a finite dimensional representation of (2.10), the identities (2.2) are trivially satisfied. For the case under inspection in this work this will not be a constraint, since the generalised Jacobi identity will be trivially satisfied as well. This happens because the four-brackets $\left\{\mathfrak{g}_{1}, \mathfrak{g}_{1}, \mathfrak{g}_{1}, \mathfrak{g}_{1}\right\}$ close upon $P_{\mu}$ or $Z$ (see below) thus we automatically have $\left[\left\{\mathfrak{g}_{1}, \mathfrak{g}_{1}, \mathfrak{g}_{1}, \mathfrak{g}_{1}\right\}, \mathfrak{g}_{1}\right]=0$. Finally, since the quadratic relations (2.8) imply the quartic relations (2.10, the superalgebra is compatible with the quartic algebra structure described by equations (2.10), meaning that any algebra of the form (2.7) satisfying the (anti)commutation relations (2.8) automatically satisfies, by construction, the relations (2.10).

This last observation has an interesting consequence. It is well known that Lie (super)algebras correspond to infinitesimal transformations and that one is able to associate finite dimensional transformations having the structure of groups and leading to the notion of Lie (super-)groups. It has been further proved that using heavy algebraic machinery [6] there is a way to associate an appropriate group to higher order Lie algebras. The benefit of the previous construction is that one can associate a group to the quartic algebra using standard procedures, and therefore avoiding complicate formal tools. This means, in particular, that this direct approach reproduces the standard quantum mechanical formalism for symmetry descriptions as presented e.g in [14] (pp. 50-55). On the other hand, it constitutes a well established fact that faithful representations of higher order algebras are infinite dimensional [4, 6], which immediately implies that matrix representations are automatically non-faithful. The compatibility of 
quadratic relations with quartic relations further means that any representation of the Lie superalgebra (2.7) will also be a (non-faithful) representation of the quartic algebra (2.10) 7 . As expected, the converse is not necessarily true.

An unsuspected consequence of the construction above is that it suggests the existence of some hidden quartic symmetries in superalgebras $t$. In general terms, this construction can be understood, in some sense, as a "square" of the graded-superalgebra (2.8). By this we specifically mean that we can naturally associate to the graded superalgebra (2.8) the quartic algebra (2.10). It is in this sense that the term "hidden quartic symmetry" appears in the usual framework of graded Lie superalgebras.

\section{Supersymmetry and quartic extensions of the Poincaré algebra}

Bearing in mind that we are mainly interested on (extended) space-time symmetries compatible with the principle of relativity and quantum mechanics, it is worthy to be inspected in detail whether higher order symmetries emerge in supersymmetry. In this section, we first recall the properties of spinor in arbitrary space-time dimensions. In the next subsection, we briefly recall the principle for the construction of supersymmetric theory in arbitrary space-time dimensions and address the question whether these algebraic structures are of the form (2.8) or (2.9). We finally show to which extent quadratic extensions of the Poincaré algebra can be naturally associated to the usual supersymmetric extensions.

\subsection{Properties of spinors in arbitrary space-time dimensions}

For our purpose it is convenient to review the main properties of spinors and Dirac- $\Gamma$ matrices in any dimensions. Introducing the tensor metric in $D$-space-time dimensions $\eta_{\mu \nu}=\operatorname{diag}(1,-1, \cdots,-1)$, the Dirac $\Gamma-$ matrices are defined by,

$$
\left\{\Gamma_{\mu}, \Gamma_{\nu}\right\}=2 \eta_{\mu \nu} .
$$

One can easily show that the Dirac matrices are complex $2^{[D / 2]} \times 2^{[D / 2]}$ matrices $([a]$ representing the integer part of $a$ ). These matrices act on Dirac spinors $\Psi_{D}$. Such spinors are complex and exist in any space-time dimensions. Furthermore, when the dimension is even, on can define the chirality matrix, $\chi=i^{[D / 2]} \Gamma_{0} \cdots \Gamma_{D-1}\left(\chi^{2}=1,\left\{\Gamma_{\mu}, \chi\right\}=0\right)$ leading to the so-called Weyl spinors $\Psi_{ \pm}=1 / 2(1 \pm \chi) \Psi_{D}$. In certain dimensions, additional specific spinors can be defined. For that purpose, we introduce a matrix $B$

I If we denote $\mathfrak{g}_{(2)}\left(\right.$ resp. $\left.\mathfrak{g}_{(4)}\right)$ the superalgebra (2.7) (resp. of the quartic algebra (2.10)) and define their associated universal enveloping algebra $\mathcal{U}\left(\mathfrak{g}_{(2)}\right)$ (resp. $\left.\mathcal{U}\left(\mathfrak{g}_{(4)}\right)\right)$-for the definition of the enveloping algebra of higher order algebras see [4, 6]-), the representations of $\mathfrak{g}_{(2)}\left(\right.$ resp. $\left.\mathfrak{g}_{(4)}\right)$ extend to representations of $\mathcal{U}\left(\mathfrak{g}_{(2)}\right)\left(\right.$ resp. $\left.\mathcal{U}\left(\mathfrak{g}_{(4)}\right)\right)$. As a consequence of the quotient $\mathcal{U}\left(\mathfrak{g}_{(4)}\right) / \mathcal{I}_{(2)} \cong \mathcal{U}\left(\mathfrak{g}_{(2)}\right)$ (where $\mathcal{I}_{(2)}$ is the two-sided ideal generated by the relations (2.8) - or (2.9) -) it turns out that a representation of $\mathfrak{g}_{(2)}$ is also a representation of $\mathfrak{g}_{(4)}$.

+ Successive attempts to adapt this methodology to the framework of colored Lie superalgebras [15, have led to serious formal obstructions that cannot be surmounted. 
such that,

$$
B \Gamma_{\mu} B^{-1}= \pm \Gamma^{\mu \star},
$$

where $\Gamma^{\star}$ denotes the complex conjugate of the matrix $\Gamma$. The sign in the equation above depends on the space-time dimension. When the matrix $B$ satisfies the property $B B^{\star}=1$, a Majorana spinor can be defined. A Majorana spinor exists in certain dimensions and satisfies the condition $\Psi^{\star}=B \Psi$. In such a case one can find a realisation of the Dirac matrices where all the matrices are real (or purely imaginary), and as a consequence the components of a Majorana spinor can be chosen to be real. On the contrary, when $B B^{\star}=-1$, an $S U(2)$-Majorana spinor $\Psi^{i}, i=1,2$ can be defined. An $S U(2)$-Majorana spinor satisfies $\Psi_{i}^{\star}=\epsilon_{i j} B \Psi^{j}$ with $\epsilon_{i j}$ the $S U(2)$ invariant volume form. The various types of spinors strongly depend on the space-time dimension. It is however important to emphasize that the results are indeed periodic of period eight. As simple consequence, a property valid in $D$-space-time dimensions is also valid in $(D+8)$-dimensions. For instance, a Majorana spinor in $D=3$ can be defined, but also in $D=11$. Table 1 summarizes the type of spinors, given modulo 8 , that is, from $D=0 \bmod 8$ to $D=7 \bmod 8$.

Table 1. Types of spinors in various dimensions, $N_{f}$ indicates the number of real components of a given spinor.

\begin{tabular}{|c|c|c|c|c|c|c|c|}
\hline$D=0 \bmod 8$ & $D=1 \bmod 8$ & $D=2 \bmod 8$ & $D=3 \bmod 8$ & $D=4 \bmod 8$ & $D=5 \bmod 8$ & $D=6 \bmod 8$ & $D=7 \bmod 8$ \\
\hline Majorana & Majorana & $\begin{array}{c}\text { Majorana- } \\
\text { Weyl }\end{array}$ & Majorana & Majorana & $\begin{array}{c}S U(2)- \\
\text { Majorana }\end{array}$ & $\begin{array}{c}\text { MUjorana- } \\
\text { Weyl }\end{array}$ & $\begin{array}{c}S U(2)- \\
\text { Majorana }\end{array}$ \\
\hline$N_{f}=2^{\frac{d}{2}}$ & $N_{f}=2^{\frac{d-1}{2}}$ & $N_{f}=\frac{1}{2} 2^{\frac{d}{2}}$ & $N_{f}=2^{\frac{d-1}{2}}$ & $N_{f}=2^{\frac{d}{2}}$ & $N_{f}=2^{\frac{d-1}{2}}$ & $N_{f}=\frac{1}{2} 2^{\frac{d-1}{2}}$ & $N_{f}=2^{\frac{d}{2}}$ \\
\hline
\end{tabular}

If we introduce $\Gamma^{\mu}$ the Dirac $\Gamma$-matrices in $D$ space-time dimensions and $C$ the charge conjugation matrix defined by

$$
C \Gamma^{\mu} C^{-1}= \pm \Gamma^{\mu t},
$$

where $\Gamma^{t}$ denotes the transpose of the matrix $\Gamma$ and the sign depends on the space-time dimension, the matrices $\Gamma^{\mu} C$ and $C$ are either symmetric or anti-symmetric depending on the dimension as indicated in Table 2 (the results are also periodic of period eight).

Table 2. Symmetry of the $\Gamma$-matrices

\begin{tabular}{|c|c|c|c|c|c|c|c|c|}
\hline$D$ mod 8 & 0 & 1 & 2 & 3 & 4 & 5 & 6 & 7 \\
\hline$C$ & sym & sym & $\begin{array}{c}\text { sym } \\
\text { anti-sym }\end{array}$ & anti-sym & anti-sym & anti-sym & $\begin{array}{c}\text { sym } \\
\text { anti-sym }\end{array}$ & sym \\
\hline$\Gamma^{\mu} C$ & $\begin{array}{c}\text { sym } \\
\text { anti-sym }\end{array}$ & sym & sym & sym & $\begin{array}{c}\text { sym } \\
\text { anti-sym }\end{array}$ & anti-sym & anti-sym & anti-sym \\
\hline
\end{tabular}


Finally, we recall that the tensor product of two Weyl spinors for $D=2 n$ decomposes on the set of $p$-forms ([p] denotes $p$-forms and $[n]_{ \pm}$(anti-)self-dual $n$-forms)

$$
\begin{aligned}
& \mathcal{S}_{+} \otimes \mathcal{S}_{+}=\left\{\begin{array}{ll}
{[0] \oplus[2] \oplus \cdots \oplus[n]_{+}} & \text {when } n \text { is even } \\
{[1] \oplus[2] \oplus \cdots \oplus[n]_{+}} & \text {when } n \text { is odd }
\end{array},\right. \\
& \mathcal{S}_{+} \otimes \mathcal{S}_{-}=\left\{\begin{array}{ll}
{[1] \oplus[3] \oplus \cdots \oplus[n-1]} & \text { when } n \text { is even } \\
{[0] \oplus[2] \oplus \cdots \oplus[n-1]} & \text { when } n \text { is odd }
\end{array},\right.
\end{aligned}
$$

with $\mathcal{S}_{ \pm}$denoting the left- (and right-)handed spinors.

These results constitute standard material and can be found in many textbooks on the subject (see e.g. [16]).

\subsection{Supersymmetry in any space-time dimensions: a brief summary}

Adjoining to the Poincaré generators spinors of the form given in Table 1, one is able to construct a supersymmetric extension of the Poincaré algebra in space-time dimensions $D \leq 11$ (see e.g. [17] for a systematic study of supersymmetry in arbitrary space-time dimensions). In order to obtain a quartic extension of the Poincaré algebra associated to supersymmetry along the lines of Section 2 , we have to check whether or not the various superalgebras are of the form (2.8) or (2.9). This means in particular that since $\mathfrak{g}_{(1,1)}=\langle Z\rangle \neq \emptyset$ - or $\mathfrak{g}_{(0,0)}=\langle Z\rangle \neq \emptyset$ - at least one central charge is needed, and thus at least an $N=2$ supersymmetry. Inspecting the $D$-dimensional supersymetric extensions of the Poincare algebra with the help of Table 2 and playing with the central charges [17, one observes that the $N=2$ supersymmetric algebra may be put on the form of (2.7) (or its modified version) in all dimensions but $D=5,11$. It turns out that the $D=8,10$ cases are exceptional since only one Majorana spinor is required. In order to illustrate the procedure we focus on the $D=10$ case. In order to reproduce the superalgebra of the form (2.8) we have to consider type IIA supersymmetry (type $I$ and IIB are excluded). Type $I I A$ supersymmetry is based on a Majorana spinor (or equivalently one left-handed and one right-handed Majorana-Weyl spinors). With the notations of (2.5) we have $\mathfrak{g}_{0}=\mathfrak{g}_{(0,0)} \oplus \mathfrak{g}_{(1,1)}=I \mathfrak{s o}(1,9) \oplus\langle Z\rangle, \mathfrak{g}_{1}=\mathfrak{g}_{(1,0)} \oplus \mathfrak{g}_{(0,1)}=\left\langle Q_{a}^{+}\right\rangle \oplus\left\langle Q_{a^{\prime}}^{-}\right\rangle$and the fermionic part of the algebra takes the form

$$
\left\{Q_{a}^{+}, Q_{b}^{+}\right\}=2 i\left(\Sigma^{\mu} C^{-+}\right)_{a b} P_{\mu}, \quad\left\{Q_{a^{\prime}}^{-}, Q_{b^{\prime}}^{-}\right\}=2 i\left(\tilde{\Sigma}^{\mu} C^{+-}\right)_{a^{\prime} b^{\prime}} P_{\mu}, \quad\left\{Q_{a}^{+}, Q_{b^{\prime}}^{-}\right\}=Z C_{a b^{\prime}}^{+-}
$$

\subsection{Quartic extension of the Poincaré algebra associated to supersymmetry}

After these considerations we are now in situation of presenting our main result. When the $N=2$ superalgebras with one central charge of the previous section can be put on the form of (2.8) (or (2.9) ), as we have seen, they enable us to propose an alternative construction of quartic algebras. Let us stress that the role of the central charge is 
essential for the argumentation. This specifically means that up to the dimensions $D=5,11$, quartic extensions of the Poincaré algebra are realisable. An amazing feature of these considerations is that super-Poincaré algebra with central charges exhibits a hidden quartic symmetry. The quartic brackets are constructed along the lines of (2.10) and it has to be mentioned that since $P_{\mu}$ and $Z$ commute with the $Q$ 's, the generalised Jacobi identity (2.2) is satisfied independently of any representations. Moreover, as already commented, these algebraic structures associated to Lie superalgebras are hybrid, partially reflecting the structure of an algebra and partially of a four-algebra.

As an illustration, we give the structure of the algebra constructed along these lines for the exceptional case $D=10$. Using the algebraic structure (2.5) with $\mathfrak{g}_{0}=\mathfrak{g}_{(0,0)} \oplus \mathfrak{g}_{(1,1)}=I \mathfrak{s o}(1,9) \oplus\langle Z\rangle, \mathfrak{g}_{1}=\mathfrak{g}_{(1,0)} \oplus \mathfrak{g}_{(0,1)}=\left\langle Q_{a}^{+}\right\rangle \oplus\left\langle Q_{a^{\prime}}^{-}\right\rangle$and the construction given in section 2.1 one obtains the following brackets (we only give the quartic brackets)

$$
\begin{aligned}
&\left\{Q_{a_{1}}^{+}, Q_{a_{2}}^{+}, Q_{a_{3}}^{+}, Q_{a_{4}}^{+}\right\}=-8\left(\left(\Sigma^{\mu} C^{-+}\right)_{a_{1} a_{2}}\left(\Sigma^{\nu} C^{-+}\right)_{a_{3} a_{4}}+\left(\Sigma^{\mu} C^{-+}\right)_{a_{1} a_{3}}\left(\Sigma^{\nu} C^{-+}\right)_{a_{2} a_{4}}\right. \\
&\left.+\left(\Sigma^{\mu} C^{-+}\right)_{a_{1} a_{4}}\left(\Sigma^{\nu} C^{-+}\right)_{a_{2} a_{3}}\right) P_{\mu} P_{\nu} \\
&\left\{Q_{a_{1}}^{+}, Q_{a_{2}}^{+}, Q_{a_{3}}^{+}, Q_{a_{4}^{\prime}}^{-}\right\}= 4 i\left(\left(\Sigma^{\mu} C^{-+}\right)_{a_{1} a_{2}} C_{a_{3} a_{4}^{\prime}}^{+-}+\left(\Sigma^{\mu} C^{-+}\right)_{a_{1} a_{3}} C_{a_{2} a_{4}^{\prime}}^{+-}+\left(\Sigma^{\mu} C^{-+}\right)_{a_{2} a_{3}} C_{a_{1} a_{4}^{\prime}}^{+-}\right) Z P_{\mu} \\
&\left\{Q_{a_{1}}^{+}, Q_{a_{2}}^{+}, Q_{a_{3}^{\prime}}^{-}, Q_{a_{4}^{\prime}}^{-}\right\}=\left(-8\left(\Sigma^{\mu} C^{-+}\right)_{a_{1} a_{2}}\left(\Sigma^{\nu} C^{+-}\right)_{a_{3}^{\prime} a_{4}^{\prime}} P_{\mu} P_{\nu}+C_{a_{1} a_{3}^{\prime}}^{+-} C_{a_{2} a_{4}^{\prime}}^{+-} Z^{2}+C_{a_{2} a_{3}^{\prime}}^{+-} C_{a_{1} a_{4}^{\prime}}^{+-} Z^{2}\right),
\end{aligned}
$$

plus similar brackets involving one $Q^{+}$and three $Q^{-}$or four $Q^{-}$. We can observe that the algebra has a very similar appearance to (2.5) (compare in particular to the second relation).

As we have mentioned previously, a representation of the super-Poincaré algebra is automatically a representation of the induced quartic algebra. Since the assumption on the non-vanishing of the central charge seems to be more interesting (look at the second bracket in (2.10) in relation to the second bracket of (2.4)), we only have considered the case were the representation of supersymmetry does not trivialise the central charge $Z$. This fact excludes the massless representations and the massive BPSsaturated bound [18]. In other words, massive representations with $|Z|<2 M$ show an unexpected behaviour under quartic symmetries. But now, since we are considering massive representations, the number of fermionic degrees of freedom cannot exceed $N_{f} \leq 16$. In consequence, the only dimensions where a quartic extension leading to non-trivial results exist are $D=2,3,4$ and 7 , respectively. The number of supercharges are such that the required spinors for the extensions are like follows:

(i) in $D=2$ one left-handed and one right-handed Majorana-Weyl spinor;

(ii) in $D=3,4$ two Majorana spinors;

(iii) in $D=7$ one $S U(2)$-Majorana spinor.

An appealing consequence of this is that the massive invariant $N=2$ Lagrangians constructed so far in this specific dimensions are moreover invariant with respect to the transformations induced by the quartic algebra. Thus, the corresponding $N=2$ supermultiplet and their associated transformations laws will automatically be an 
invariant multiplet of the corresponding quartic structure with the same transformation properties (see footnote 9 on page 6). Therefore, this simple observation means that, within this construction, interacting Lagrangians invariant under quartic symmetries are obtained. In the same sense as the notion of supersymmetry surmounts the obstruction described by the no-go theorem of Coleman-Mandula [19], our present construction can be seen as an analogous expansion that avoids the constraints of the Haag-LopuszanskiSohnius theorem [20]. This analogy should however not be misunderstood at the conceptual level. While supersymmetry represents a fertile novelty (with respect to classical inner/outer symmetry analysis) by means of the introduction of fermionic charges, therefore leading to new phenomenological aspects, the construction of quartic algebras executed in this work is heavily dependent on the supersymmetric algebra formalism and underlying constraints.

\subsection{Representations of quartic extensions}

The ansatz linking algebras of order four to Lie superalgebras has remarkable consequences concerning their respective representation theories, in the sense that superalgebra representations automatically induce representations of the order four structures. In full generality, the reversal of this assertion is not true, as we briefly justify. Consider for instance the four-dimensional quartic extensions of the Poincaré algebra in four space-time dimensions. If we study massive representations, the little algebra is generated by the $Q$ 's and $P^{0}=-i m$ and the four-brackets take the form

$$
\begin{aligned}
\left\{Q_{\alpha_{1}}{ }^{I_{1}}, Q_{\alpha_{2}}{ }^{I_{2}}, Q_{\alpha_{3}}{ }^{I_{3}}, Q_{\alpha_{4}}{ }^{I_{4}}\right\}= & 2 Z^{2}\left(\varepsilon_{\alpha_{1} \alpha_{2}} \varepsilon_{\alpha_{3} \alpha_{4}} \varepsilon^{I_{1} I_{2}} \varepsilon^{I_{3} I_{4}}\right. \\
& \left.+\varepsilon_{\alpha_{1} \alpha_{3}} \varepsilon_{\alpha_{2} \alpha_{4}} \varepsilon^{I_{1} I_{3}} \varepsilon^{I_{2} I_{4}}+\varepsilon_{\alpha_{1} \alpha_{4}} \varepsilon_{\alpha_{2} \alpha_{3}} \varepsilon^{I_{1} I_{4}} \varepsilon^{I_{2} I_{3}}\right), \\
\left\{Q^{I_{1}}{ }_{\alpha_{1}}, Q^{I_{2}}{ }_{\alpha_{2}}, Q^{I_{3}}{ }_{\alpha_{3}}, \bar{Q}_{I_{4} \dot{\alpha}_{4}}\right\}= & 2 m Z\left(\delta^{I_{1}}{ }_{I_{4}} \varepsilon^{I_{2} I_{3}} \varepsilon_{\alpha_{2} \alpha_{3}} \sigma^{0}{ }_{\alpha_{1} \dot{\alpha}_{4}}+\delta^{I_{2}}{ }_{I_{4}} \varepsilon^{I_{1} I_{3}} \varepsilon_{\alpha_{1} \alpha_{3}} \sigma_{\alpha_{2} \dot{\alpha}_{4}}^{0}\right. \\
+ & \left.\delta^{I_{3}}{ }_{I_{4}} \varepsilon^{I_{1} I_{2}} \varepsilon_{\alpha_{1} \alpha_{2}} \sigma^{0}{ }_{\alpha_{3} \dot{\alpha}_{4}}\right), \\
\left\{Q^{I_{1}}{ }_{\alpha_{1}}, Q^{I_{2}}{ }_{\alpha_{2}}, \bar{Q}_{I_{3} \dot{\alpha}_{3}}, \bar{Q}_{I_{4} \dot{\alpha}_{4}}\right\}= & 2 m^{2}\left(\delta^{I_{1}}{ }_{I_{3}} \delta^{I_{2}}{ }_{I_{4}} \sigma^{0}{ }_{\alpha_{1} \dot{\alpha}_{3}} \sigma_{\alpha_{2} \dot{\alpha}_{4}}^{0}+\delta^{I_{1}}{ }_{I_{4}} \delta^{I_{2}}{ }_{I_{3}} \sigma_{\alpha_{1} \dot{\alpha}_{4}}^{0} \sigma_{\alpha_{2} \dot{\alpha}_{3}}^{0}\right) \\
& +2 Z^{2} \varepsilon_{\alpha_{1} \alpha_{2}} \varepsilon_{\dot{\alpha}_{3} \dot{\alpha}_{4}} \varepsilon^{I_{1} I_{2}} \varepsilon_{I_{3} I_{4} .} .
\end{aligned}
$$

If we now make the following substitutions (analogous to the corresponding substitution for the $N=2$ supersymmetric extension with central charge):

$$
\begin{array}{ll}
a^{1}=Q^{1}{ }_{1}-\bar{Q}_{2 \dot{2}}, & a^{3}=Q^{1}{ }_{1}+\bar{Q}_{2 \dot{2}}, \\
a^{2}=Q^{1}{ }_{2}+\bar{Q}_{2 \dot{1}}, & a^{4}=Q^{1}{ }_{2}-\bar{Q}_{2 \dot{1}}
\end{array}
$$

one observes that $a^{1}, \cdots, a^{4}, a_{1}^{\dagger}, \cdots, a_{4}^{\dagger}$ generate the Clifford algebra of the polynomial

$$
\begin{aligned}
P^{2}\left(x_{1}, \cdots, x_{4}, y^{1}, \cdots, y^{4}\right)= & \left(2(2 m+Z) x_{1} y^{1}+2(2 m+Z) x_{2} y^{2}+2(2 m-Z) x_{3} y^{3}\right. \\
& \left.+2(2 m-Z) x_{4} y^{4}\right)^{2}
\end{aligned}
$$

in the sense that

$$
\left(x_{I} a^{I}+y^{I} a_{I}^{\dagger}\right)^{4}=P^{2}\left(x_{1}, \cdots, x_{4}, y^{1}, \cdots, y^{4}\right) .
$$


The representations of the $N=2$ supersymmetric algebra in four dimensions are obtained from the study of representations of the Clifford algebra, i.e. when the $a$ 's satisfy the quadratic relation,

$$
\left(x_{I} a^{I}+y^{I} a_{I}^{\dagger}\right)^{2}=P\left(x_{1}, \cdots, x_{4}, y^{1}, \cdots, y^{4}\right),
$$

which is obviously compatible with (3.7). On the contrary, one can construct representations of (3.7) such that (3.8) is not satisfied. The algebra (3.7) has been introduced a long time ago by mathematicians and is called the Clifford algebra of the polynomial $P^{2}$ [21]. It has been shown that to any polynomial $f$ one can associate a Clifford algebra $\mathcal{C}_{f}$, and that a matrix representation can be obtained [22]. But for polynomials of degree higher than two, the representation is not unique, and various inequivalent representations of $\mathcal{C}_{f}$ (even of the same dimension) can be constructed (see, for instance, [23]). This difficulty contributes considerably to the problem of classifying representations of $\mathcal{C}_{f}$, which is still open, though it has been proved that the dimension of the representation is a multiple of the degree of the polynomial [24].

In the framework of our analysis, this may provide new representations corresponding to interesting quartic extensions of the Poincaré algebra. This hierarchy of representations on the top of the standard representations obtained in supersymmetric theories might be compared to the parafermionic extension of the Poincaré algebra considered in 25]. It turns out that the algebra studied there shares some similarities with ours, like the non-linearity and the possibility of deriving a hierarchy of representations starting from the standard supersymmetric one. To which extent these two different approaches have additional far-reaching common features and reflect physical phenomena in supersymmetric theories still constitutes work in progress.

\section{Perspectives and outlook}

In this paper we have given a systematic way to associate a quartic Lie algebra (which closes with fully symmetric quartic brackets) to a graded Lie superalgebra of a certain type. In particular, this construction can be applied to standard supersymmetric theories. This specifically alludes to the fact that any representation of $N=2$ supersymmetric algebras shares a hidden quartic symmetry. We insist upon the fundamental role of the non-vanishing central charge, implying that, in these conditions, any supersymmetry Lagrangian reflects a hidden quartic symmetry. One may wonder whether this apparently simple but unexpected peculiarity is a consequence of some deep "raison d'être" responding to some structural or phenomenological aspect adequately described by this type of hidden symmetry, and somehow encoded in the conformation of the Lagrangian.

Another question that emerges naturally from our analysis is what happens if we relax the assumption on the non-vanishing of the central charge. This opens the possibility of having massless representations, and we are no more limited to the few cases exhibited in section 3. A particularly interesting case is that of ten-dimensional 
type II A supersymmetry, which constitutes in some sense an exceptional model - recall that in this case a quartic extension is constructed with only one Majorana spinor and inherits a hidden quartic symmetry in quite natural way.

We have pointed out that the construction of the quartic algebra structure does not impose constraints on the number of central charges. In this sense, an analogous analysis of $N>2$ supersymmetric extensions can be considered. Also in this case, the quartic Clifford algebras enable us to connect the underlying representation theory of these structures. The main difficulty in this task refers to the fact whether the $N>2$ extensions can be written in the adequate form, in order to apply the procedure, as well as to consider the physically relevant models. Work in this direction is currently in progress.

\section{Acknowledgments}

During the preparation of this work, one of the authors (RCS) was financially supported by the research project GR58/4120818-920920 of the UCM-BSCH.

\section{References}

[1] Nambu Y 1973 Phys. Rev. D 72405

Curtright T and Zachos C K 2003 Phys. Rev. D 68085001 arXiv:hep-th/0212267.

[2] Bagger J and Lambert N 2008 Phys. Rev. D 7, 065008 [arXiv:0711.0955 [hep-th]].

Bagger J and Lambert N 2009 Phys. Rev. D 79025002 [arXiv:0807.0163 [hep-th]].

Gustavsson A 2009 Nucl. Phys. B 81166 arXiv:0709.1260 [hep-th]].

[3] Azcárraga J A de and Izquierdo J M 2010 J. Phys. A: Math. Theor. 43293001 [arXiv:math$\mathrm{ph} / 1005.1028 \mathrm{v} 1]$

[4] Rausch de Traubenberg M and Slupinski M J 2000 J. Math. Phys. 414556 arXiv:hep-th/9904126. Rausch de Traubenberg M and Slupinski M J 2002 J. Math. Phys. 435145 arXiv:hep-th/0205113.

[5] Goze M, Rausch de Traubenberg M and Tanasa A 2007 J. Math. Phys. 48093507 arXiv:math-ph/0603008.

[6] Rausch de Traubenberg M 2008 J. Phys. Conf. Ser. 128012060 arXiv:0710.5368 [math-ph]];

Goze M and Rausch de Traubenberg M 2009 J. Math. Phys. 50063508 arXiv:0809.4212 [math$\mathrm{ph}]$.

[7] Campoamor-Stursberg R and Rausch de Traubenberg M 2009 J. Gen. Lie Theory Appl. 3113 arXiv:0811.3076 [math-ph]].

[8] Mohammedi N, Moultaka G and Rausch de Traubenberg M 2004 Int. J. Mod. Phys. A19 5585 arXiv:hep-th/0305172.

[9] Moultaka G, Rausch de Traubenberg M and Tanasa A 2005 Int. J. Mod. Phys. A20 5779 arXiv:hep-th/0411198.

[10] Moultaka G, Rausch de Traubenberg M and Tanasa A 2004, in Proceedings of the XIth International Conference Symmetry Methods in Physics, Prague 21-24 June 2004 arXiv:hep-th/0407168.

[11] Campoamor-Stursberg R and Rausch de Traubenberg M 2009, J. Phys. A; Math. Theor. 42 495202 arXiv:0907.2149 [hep-th]].

[12] Filippov V 1968 Sibirsk. Mat. Zh. 39660

[13] Figueroa-O'Farrill J 2009 J. Phys. A: Math. Theor. 42, 445206 arXiv:0905.4900 [hep-th]]. 
[14] Weinberg S 1995 The Quantum Theory of Fields. Vol. 1: Foundations, (Cambridge, UK: Univ. Pr. )

[15] Lukierski J and Rittenberg V 1978 Phys. Rev. D 18385

[16] Rausch de Traubenberg M 2009 Adv. Appl. Cliff. Algebra 19269 arXiv:hep-th/0506011.

[17] Strathdee J A 1987 Int. J. Mod. Phys. A 2273

[18] Ferrara S, Savoy C A and Zumino B 1981 Phys. Lett. B 100393

[19] Coleman S and Mandula J 1967 Phys. Rev. 1591251

[20] Haag R, Lopuszanski J T and Sohnius M F 1975 Nucl. Phys. B88 257

[21] Roby N 1969 C. R. Acad. Sc. Paris 268484

Roby N 1970 Bull. Sc. Math. 9449

Revoy Ph 1977 C. R. Acad. Sc. Paris 284985

[22] Fleury N and Rausch de Traubenberg M 1992 J. Math. Phys. 333356

Fleury N and Rausch de Traubenberg M 1994 Adv. Appl. Cliff. Alg. 4123

[23] Revoy Ph 1993 Adv. Appl. Cliff. Alg. 339

[24] Haile D H and Tesser S 1988 J. Alg. 116372

[25] Jarvis P D 1978 Aust. J. of Phys. 31461 